\title{
Utterance ambiguity in uncertainty contexts strengthens scalar implicature ${ }^{1}$
}

\author{
Izabela Skoczeń $^{1}$ and Aleksander Smywinski-Pohl ${ }^{2}$ \\ ${ }^{1}$ Jagiellonian University \\ ${ }^{2}$ AGH University of Technology
}

\begin{abstract}
N. Goodman and A. Stuhlmüller's experiment provides empirical support for the Rational Speech Act (RSA) model that predicts the probability of implicature inference in uncertainty contexts. We present a partial replication of this experiment in English and Polish; we argue that the partial replication indicates a possible ambiguity in the experimental design concerning the 'some' quantifier term. We verify the ambiguity of the quantifier term hypothesis in a redesigned experiment and argue that our results support the idea that the RSA model encodes a robust theory of communication.
\end{abstract}

Keywords: scalar implicature, incomplete knowledge, rational speech act model, semantic-pragmatic interface, quantifier term.

\section{Introduction}

How do our beliefs about what a speaker meant interfere with our beliefs about the state of the world? Proponents of the existence of a separate, linguistic module for pragmatic computations suggest that there may be such interference yet it is not systematic (Fodor 1983). Relevance theorists claim that utterance understanding draws on world knowledge and is carried out by a dedicated but not informationally encapsulated module that operates in accordance with the relevance heuristic (Sperber and Wilson 2002). Moreover, middle-ground accounts which postulate that a good deal of these mechanisms are default or encoded in the grammar of a language claim that there is such interference yet do not provide an account of its mechanisms (Genaro Chierchia et al. 2011). By contrast, the Rational Speech Act (RSA) theory claims a systematic interaction between utterance understanding and world knowledge shared by interlocutors. RSA views language understanding as a special case of a broader mechanism, namely social cognition. Based on the assumptions of RSA, Bayesian decision theory and game theory, a model of human behavior was created (Frank and Goodman 2012).

Noah Goodman and Andreas Stuhlmüller carried an experimental study that provided empirical support for the claim that the RSA model provides robust predictions concerning the formation of listeners' beliefs about the world in varied contexts (Goodman and Stuhlmüller 2013).

The main aim of the present paper is a replication of the experiment in question in both the English and Polish language. This could provide an answer to a different question: whether the semantic-pragmatic interface of the English language that is encoded in the discussed version of the RSA model described in N. Goodman and A. Stuhlmüller's paper holds cross-linguistically. If it is confined to the English language, then modeling different languages might require different formalisms in the RSA model. The experiment designed by N. Goodman and A. Stuhlmüller focuses on a particular type of pragmatic inference, namely scalar implicature.

\subsection{Scalar implicatures}

Scalar implicatures are special because they are based on conventional meanings attributed to words with the use of lexical scales (Horn 2006). If you hear:

\footnotetext{
${ }^{1}$ The research was funded by [removed for anonymity purposes]. The Authors would like to thank [removed for anonymity purposes]. Appendix, all datasets and preregistrations are available in the following repository: https://osf.io/agbfd/?view_only=b59738d84dba49a79d28bfc60865067e .
} 
(I.) Some of the students passed the exam.

you infer that not all of them passed. In complete knowledge situations, your reasoning is as follows: had the speaker known that all of the students passed the exam, she would have used a stronger word on the scale, namely the word 'all.' Since she said 'some' (a weaker word on the scale), then not all of the students passed the exam.

A lexical scale is defined as a 'totally ordered set of lexical items which vary along a single dimension' (Horn 1972) (Bergen et al. 2016, p. 10). Placing a word in a position on a scale enables speakers to attribute to it a meaning that does not have to be consistent with the lexical meaning of the word. The most discussed example in the literature is indeed the quantifier term 'some,' which can mean either 'at least some' or 'some but not all.' In ordinary conversation, 'some' is most often understood as 'some but not all.' This is valid when the speaker and hearer have full knowledge of the context. This means that, for example, the speaker knows exactly how many of the guests left the party. She knows whether all of them have gone or only a number of them have gone. Moreover, the hearer knows that the speaker has this knowledge. Consequently, suppose that the speaker says:

(II.) Some of the guests have left the party.

In this case, the hearer infers that not all of the guests have left, otherwise the speaker would have used the word 'all.' This is labeled a 'knowledge implicature' (Bergen and Grodner 2012). 'The stronger term (all) implies the weaker term (some), but the weaker term (some) implicates the negation of the stronger term (some = not all)' (de Carvalho et al. 2016). Thus, the lexical scale is the following:

(III.) $<$ some, all >

However, if the speaker does not know whether some or all of the guests have left the party and the hearer knows this, the interpretation changes. Now, the hearer infers that 'at least some' of the guests have left the party. This is labeled an 'uncertainty implicature' (Bergen and Grodner 2012). As a result, according to the described account, 'at least some or some but not all' is taken to be the meaning of 'some.'

The distinction between 'knowledge implicatures' and 'uncertainty implicatures' implies that not inferring a scalar implicature and cancelling a scalar implicature are two distinct processes. You can only cancel a knowledge implicature. If the speaker has full knowledge of a context and says:

(IV.) Some of the guests left the party, in fact, all of them did.

Then the hearer first infers 'some but not all of the guests left' and only subsequently cancels this inference upon hearing the second part of the utterance. By contrast, the so-called uncertainty implicatures consist in completely abstaining from the scalar inference 'some but not all.' This distinction finds empirical support in experimental studies that measured participants' reaction times. Tasks involving a cancellation of a scalar knowledge implicature generated longer answering times in participants, while uncertainty implicatures tasks generate quicker answering times (Bott and Noveck, 2004, Noveck and Posada, 2003, Noveck and Sperber, 2004). The RSA model described in Goodman and Stuhlmüller's 2013 paper does not distinguish between cancelling and refraining from inferring scalar implicatures; 
rather, this iterative model predicts that in a context of partial access or uncertainty, the scalar implicature is not formed in subsequent iterations. Since time measures were not discussed in the study at stake, we remain agnostic on the issue throughout the paper. However, we believe that the Authors of the study we are replicating, namely (Goodman and Stuhlmüller 2013), are not fully warranted in their employment of the word 'cancellation' of the scalar implicature. For this reason, in the description of our results we decided to employ the terminology that an implicature 'does not occur' rather than that it is 'canceled.'

There is an ongoing heated debate on the nature of scalar implicatures. Broadly speaking, there are three main camps: the grammatical, the lexical and the pragmatic (Sauerland 2012). The first claims that scalars are generated by mechanisms encoded in the grammar; they postulate the existence of a silent operator labeled Exhaustify or Only which operates on the set of alternatives delimited by the lexical scale (Gennaro Chierchia 2006), (Fox 2007), (Genaro Chierchia et al. 2011).

The second camp claims that scalar terms are governed by purely lexical mechanisms. In other words, they are part of the lexicon that can take pragmatically modulated word meanings. The idea is that the pragmatic interpretation (some but not all) is lexicalized as the default interpretation. "The pragmatic interpretation of scalar items is encoded as a (defeasible) part of its meaning (i.e., "some" also means "not all"), while the semantic interpretation (i.e., "some = at least one") would only be accessible if the pragmatic interpretation is explicitly negated (e.g., "The pianist played some Mozart sonatas and even all of them")' (de Carvalho et al. 2016, p. 2) 2

Lastly, the third camp claims that scalar implicatures arise due to purely pragmatic mechanisms. There are two main sub-camps within the pragmatic view: The first is the explanation provided by Relevance Theory (RT), which claims that scalar implicatures are explicatures which are pragmatic developments of the logical form of a sentence (Sperber and Wilson 2006). The logical form or the linguistic interpretation of a sentence is pragmatically enriched to obtain a proposition with the relevant truth conditions. The final stage of the process is an ad hoc concept construction that can take the form of two processes: loosening or strengthening. For example, if someone says at a restaurant that a steak is raw, then he means that the steak is merely undercooked. According to RT, scalar implicatures involve ad hoc concepts that are generated without the use of lexical scales. Moreover, RT predicts that scalar interpretations are cognitively costly because they always involve fine-grained contextual inference (Sperber and Wilson, 2006, de Carvalho et al., 2016). Experimental work confirm that scalars are cognitively costly in the sense that children start using them only in later stages of development. (Noveck, 2001, Papafragou and Musolino, 2003, Papafragou and Tantalou, 2004, Guasti et al., 2005 Pouscoulous et al., 2007).

However, there is some processing evidence for the existence of lexical scales. This means that lexical scales can mitigate the cognitive cost of scalar derivation; see for instance the priming experiments done by (de Carvalho et al. 2016). These experiments indicate that there is an interpretive asymmetry in a lexical scale, namely, 'stronger words are necessary for the interpretation of weaker words, but the reverse is not true' (de Carvalho et al. 2016). Consequently, there is an asymmetry in priming: 'weaker words in a scale (...) prime stronger words in the same scale more than stronger words (...) prime weaker words. For instance, in the scale <all, many, some>, some (...) primes many and all more than many would prime some and more than all would prime many and some' (de Carvalho et al. 2016).

\footnotetext{
${ }^{2}$ This account explains best local scalar implicatures, but has issues in explaining global scalar implicatures based on entire speech acts (Levinson, 2000, Chierchia, 2004).
} 
The second sub-camp within the pragmatic view explains scalars through the hearer's reasoning about why the speaker chose a particular utterance in the circumstances and restricts the possible set of alternative utterances in line with the question under discussion. Moreover, the hearer reasons globally about entire speech acts (Horn, 1972, Atlas and Levinson, 1981, Spector, 2003, Sauerland, 2004, Russell, 2006, Geurts, 2010, and others). The RSA framework discussed in the present paper models scalar inferences as pragmatic and implements lexical scales into a game-theoretic setting (Bergen et al. 2016).

In Polish, the word 'niektóre' is the counterpart of the quantificational uses of the English word 'some' (see for instance Piasecki, 2002). 'Niektóre' can mean either 'przynajmniej niektóre' (at least some) or 'tylko niektóre' (some but not all). Moreover, in an ordinary conversation 'niektóre' is understood as 'tylko niektóre' (some but not all) and this inference is contextually cancellable. However, just as the French counterpart of the quantifier term 'some' is 'certains de' (see for instance Pouscoulous et al., 2007), the Polish 'niektóre' is plural in the sense that it is used to denote at least two objects, contrary to the word 'some,' which can be used to refer to a single object. Thus, while logicians are fully warranted to label the English 'some' as a linguistic counterpart of existential quantification leading to the inference that there exists at least one object that has a property, this inference is not warranted as far as the Polish word 'niektóre' is concerned. ${ }^{3}$

Let us now proceed to the RSA model of scalar implicatures presented and experimentally supported in (Goodman and Stuhlmüller 2013), whose empirical results we attempted to replicate in the English and Polish languages.

\subsection{The Rational Speech Act model of scalar implicatures}

Noah Goodman and Andreas Stuhlmüller model utterance understanding in scenarios in which the speaker has partial contextual knowledge and the hearer is informed that the speaker has partial contextual knowledge.

The model is a particular version of the more general 'rational speech act model (RSA)' (Frank and Goodman, 2012, Goodman and Frank, 2016, Degen et al., n.d.). This model views linguistic inference as a special case of rational inference. The hearer is modeled as an agent reasoning about the state of the world $s$ on the basis of the utterance $w$ he has heard as well as the access $a$ which captures the limited knowledge of context, namely, 'shared information about the speaker's (possibly incomplete) information access' (Goodman and Stuhlmüller 2013). Using Bayes' rule, the hearer model is proportional to:

Plistener $(\mathrm{s} \mid \mathrm{w}, \mathrm{a}) \propto \operatorname{Pspeaker}(\mathrm{w} \mid \mathrm{s}, \mathrm{a}) \mathrm{P}(\mathrm{s})$

\footnotetext{
${ }^{3}$ Moreover, there are differences between the remaining (non-quantificational) possible uses of the English 'some' and the Polish word 'niektóre.' Namely, the English 'some' can be used as a singular or plural indefinite in the following sentences:

(I.) This is just some book that I bought last week.

(II.) Yesterday I went to the store and I bought some books.

By contrast, the Polish translation of the sentences above could not include the word 'niektóre.' Polish users would rather employ words such as 'jakieś' or 'pewne.' A similar pattern is exhibited by the Russian language (Honselaar, 1983, Zinova and Filip, n.d.).
} 
Where $\mathrm{P}(\mathrm{s})$ is the hearer's prior probability distribution on possible world states and $\mathrm{P}_{\text {speaker }}(\mathrm{w} \mid \mathrm{s}, \mathrm{a})$ describes the listener's theory about how the speaker chooses utterances' (Goodman and Stuhlmüller 2013, p. 175).

A speaker reasons about which utterance $w$ she should choose on the basis of the observation $o$ she made and her access. She does so by 'soft-max optimizing the expected utility,' the speaker chooses utterances in (soft-max) proportion to the information they convey; as the soft-max approaches infinity, she will choose the maximally informative utterance $100 \%$ of the time; as soft-max goes to 1 , she will choose utterances in proportion to the informativity:

$$
\mathrm{P}_{\text {speaker }}(\mathrm{w} \mid \mathrm{o}, \mathrm{a}) \propto \exp (\alpha \mathrm{E} \sim \mathrm{P}(\mathrm{s} \mid \mathbf{o}, \mathrm{a})[\mathrm{U}(\mathrm{w} ; \mathrm{s})])
$$

$\mathrm{U}(\mathrm{w} ; \mathrm{s})$ is a utility function of the speaker which 'captures the value of saying $w$ if the world is actually $s$. The expectation ranges over the speaker's belief state, $\mathrm{P}(\mathrm{s} \mid \mathbf{0}, \mathrm{a})$, because the speaker may still be uncertain about the state of the world. The parameter controls the deviation from optimality' (Goodman and Stuhlmüller 2013, p. 175).

The utility value captures how much information is conveyed by an utterance. 'More specifically, utility is related to the amount of information that a literal listener would not yet know about state $s$ after hearing it described by utterance $w$ (the negative surprisal):

$$
\mathrm{U}(\mathrm{w} ; \mathrm{s})=\ln \left(\mathrm{P}_{\text {lex }}(\mathrm{s} \mid \mathrm{w})\right)
$$

'Where the literal interpretation probability $\mathrm{P}_{\text {lex }}(\mathrm{s} \mid \mathrm{w})$ is determined by the lexicon - here we will assume that each utterance has a truth function, Fw;s $\rightarrow\{0,1\}$, and the distribution is otherwise uninformative: $\mathrm{P}_{\text {lex }}(\mathrm{s} \mid \mathrm{w}) \propto \delta \mathrm{Fw}(\mathrm{s})$.' (Goodman and Stuhlmüller 2013, p. 175).

The speaker's access $a$ is common knowledge of both speaker and listener, but the listener still does not know what observation the speaker made:

$$
\mathrm{P}_{\text {speaker }}(\mathrm{w} \mid \mathrm{s}, \mathrm{a})=\mathrm{oP} \text { speaker }(\mathrm{w} \mid \mathrm{o}, \mathrm{a}) \mathrm{P}(\mathrm{o} \mid \mathrm{a}, \mathrm{s})
$$

For this reason, the hearer is summing over the possible observations made by the speaker (Goodman and Stuhlmüller 2013, p. 175).

The model takes the 'question under discussion' as 'how many letters have cheques in them?'. This question is a projection from the state space onto a lower dimensional representation of the state space cf. (Hawkins and Goodman forthcoming) for details.

\subsection{The experimental design}

This model has been empirically tested and confirmed in the English language (Goodman and Stuhlmüller 2013). To grasp the experimental setting, imagine a speaker that sees, for example, three apples; however, he has information concerning the color of only two of these apples. The hearer is aware of the speaker's state of knowledge. According to the conclusions of the original paper, the utterance 'some of the apples are red' did not give rise to the implicature 'not all' (or gave rise to a much weaker implicature) since hearers inferred a similar probability of two or three apples being red. If the speaker saw two out of three apples and said, 'one of the apples is red,' then the hearer inferred a similar probability of one or two of the apples being red and a low probability of three apples being red. Thus, the implicature 'not more than one' did not occur. During the experiment, six scenarios were used and the speaker had varied access to the objects and their features, while the hearer was aware of the speaker's state of knowledge. There were two versions of the experiment: with the quantifier term 'some' and with numerals. The original results from the version of the experiment with the quantifier 'some' are presented in Figure 2. Just as in the original experiment, data has been filtered to 
include only trials where the participant's bet (sum placed from the 100 units at the participants disposal) that the speaker had complete knowledge was greater than 70 in the expected direction. This means that if the speaker had complete knowledge, the participant had to bet more than 70 on the positive answer to the control question: 'do you think the speaker knows exactly how many of the objects have the relevant property?'. Error bars were standard errors of the mean. (Goodman and Stuhlmüller 2013) "Some" access 1 was the partial-access condition where the speaker has access to one object and utters some. "Some" access 2 was the partial-access condition where the speaker has access to two objects and utters some. "Some" access 3 was the total access condition where the speaker has access to three objects and utters some.

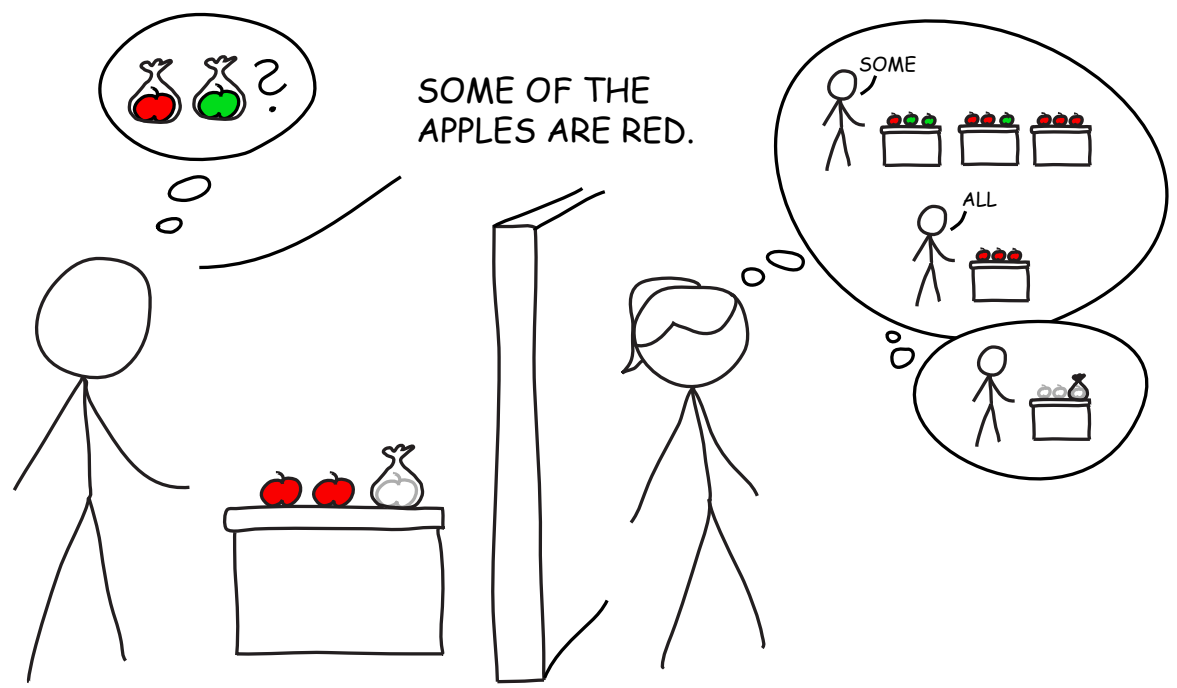

Figure 1 The experimental design; reuse license number 4796380796837

These experimental results lead to the conclusion that there is a fine-grained interaction between the state of knowledge about the world and pragmatic inference. In contexts in which uncertainty about the state of the world is high, people are reluctant to perform pragmatic inference ${ }^{4}$.

However, the remaining interesting question raised by Goodman and Stuhlmüller's results is whether the effects would be cross-linguistic or rather confined to the semantic-pragmatic interface of English. In order to check this, we decided to replicate the initial results in English to be able to compare the data with the results gathered in Polish. We choose Polish because, as described above, the semantics of the counterpart of 'some' is slightly different. Thus, if the experiment replicates also in polish, ti strengthens the claim that the model is robust. Since in Polish 'niektóre' is only true of plurals (so there must be at least 2 letters with cheques to say "niektóre letters have cheques") we predict that in the "some" access 2 condition, participants will bet much less on 1 of the letters having cheques than their English counterparts. This could be expected for the "some" access 1 condition as well, but then participants might also be confused

\footnotetext{
${ }^{4}$ The results also provided evidence against some modular theories of scalars since the data showed a systematic interference between beliefs about what the speaker meant and beliefs about the state of the world. This does not provide evidence against Relevance Theory as a modular theory, since this theory claims a built-in interference between beliefs about what the speaker meant and beliefs about the state of the world. (Sperber and Wilson, 2002)

6
} 
in this condition, because the speaker has not seen 2 (i.e., if I opened 1 letter, it would be odd for me to say " 2 letters have cheques" unless there is something that I'm not telling you).

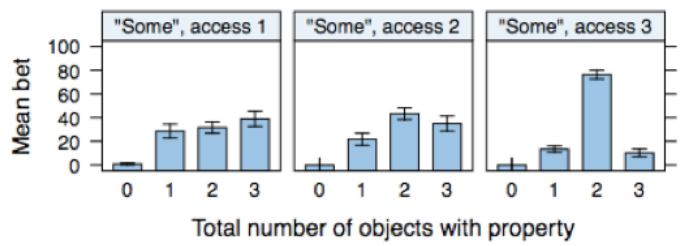

Figure 2 Data from the original experiment (Goodman and Stuhlmüller 2013); reuse license number 4796380796837

Below we present the replication of the afore-described experiment in English and Polish. We translated the same scenarios into Polish (cf. Appendix ${ }^{5}$ ). We employed the Polish words 'niektóre' for 'some, 'wszystkie' for 'all'. Our replication is partial and we argue this is due to an ambiguity in the experimental design as to the quantifier term 'some' (a contextual under specification of quantifier domain restriction $)^{6}$.

\section{$2 \quad$ Experiment 1 - Replication in English (scenario employing the term 'some')}

Before proceeding to the replication in Polish, we first performed a limited replication of the experiment in English; namely, we replicated the version of the experiment which employs the quantifier term 'some.' This is labeled 'Experiment 1 ' in (Goodman and Stuhlmüller 2013). We did this because of the semantic differences between 'some' and 'niektóre,' which we hypothesized to be critical for replication in a different language. Since Polish speakers employ the word 'niektóre' to denote at least two objects, while 'some' can be used to denote one object, we decided to check whether the participants' responses on the question on the a posteriori (ex post, after hearing utterance) probability distribution would differ across languages.

\subsection{Participants}

Just as in the original experiment, 61 participants were recruited online via Amazon Mechanical Turk. The IP address location was restricted to the USA (female: $38 \%$; mean age: 38 years, $S D=10.54$, age range: $25-65$ years). Since this is a per trial statistic (just as in the original experiment) each participant had three, within subjects, trials (183 trials in total) and we excluded trials for which participants failed a comprehension question, leaving a sample of 110 trials.

5 Appendix, all datasets and preregistrations are available in the following repository: https://osf.io/agbfd/?view_only=b59738d84dba49a79d28bfc60865067e .

${ }^{6}$ This ambiguity is rather a quantifier term ambiguity, not a quantifier scope ambiguity. This is because quantifier scope enters when there are multiple quantifiers, such as in the context of sentences like "Every apple isn't red" which can be parsed according to two relative scopes of the negation operator within the logical form: (i) *none of the apples are not* (for_all [ not ]) or (ii) *not all of the apples are red* (not [for_all]) (Montague 1973). The phenomena being explored here is about implicit domain restriction e.g., "Everyone detests Bill" probably should not be taken to mean that *all human beings* detest Bill but perhaps something more like *all human beings who know Bill detest him*(Von Fintel 1994). We thank a reviewer for this remark. 
Just as in the original experiment, we used the same 6 scenarios: letters with cheques inside, students taking an exam, fruits with dried pith, mobile phones with broken transistors, sprouting seeds and winning lottery tickets. Just as in the original study, the study began with two warm-up control questions that checked the general attention level of the participants and familiarized them with the betting measure. At the beginning of each scenario, the prior (ex ante) probability that all the objects had the salient property was boosted, for example:

Letters to Laura almost always have cheques inside (Goodman and Stuhlmüller 2013, p. 178).

Next, the information about the number of considered objects and a question about the prior probability distribution was displayed:

Today Laura received three letters. How many of the 3 letters do you think have cheques inside? (Goodman and Stuhlmüller 2013, p. 178)

This is because without this question 'it was difficult to distinguish an implicature from a prior belief that it was unlikely for all objects to have the property' (Goodman and Stuhlmüller 2013). Each participant had a sum of a hundred dollars, which she divided (through betting) between four options: zero, one, two or three objects that had the property in question. After capturing the prior probability distribution, we displayed the information about the number of objects verified by the speaker. This number varied from one to three:

Laura tells you on the phone: "I have looked at 2 of the 3 letters. Some of the letters have cheques inside" (Goodman and Stuhlmüller 2013, p. 178).

Next, a question about the posterior probability distribution followed:

Now how many of the 3 letters do you think have cheques inside? (Goodman and Stuhlmüller 2013, p. 178)

Finally, a control question was posed:

Do you think Laura knows exactly how many of the 3 letters have cheques inside? (Goodman and Stuhlmüller 2013, p. 178)

The final control question had only two betting options (knows/does not know). If a participant answered that Laura did know exactly how many letters had cheques inside, even though she verified only two letters, the data was rejected. This was done because this was interpreted as evidence that the participant was either inattentive (did not comprehend the scenario), did not trust what Laura said, or performed reasoning about the state of the world without taking into account the linguistically conveyed information. This last possibility was of no interest since the aim of the experiment was to investigate interactions between information about the world and linguistic information.

Since the speaker's access varied - for instance she verified one, two or three letters - each participant was presented with each access condition only once in a random order with randomly chosen scenarios (participants never saw the same scenario twice). Just as in the original experiment, there were two partial-knowledge conditions (without implicature) and one complete-knowledge "control" (with implicature) (Goodman and Stuhlmüller 2013). The experiment we ran can be accessed at http://apohllo.pl/psycho/index-en.html. 
80

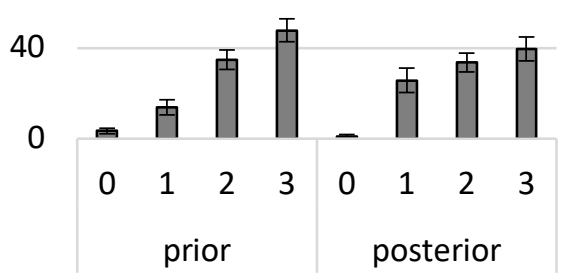

$\square$ some access 1

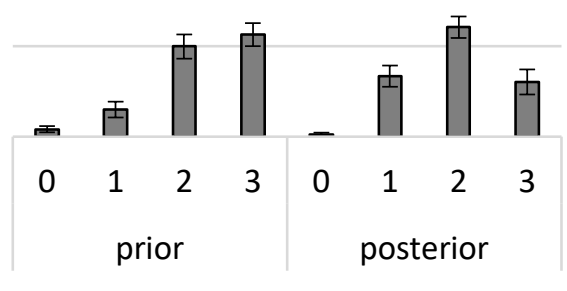

$\square$ some access 2

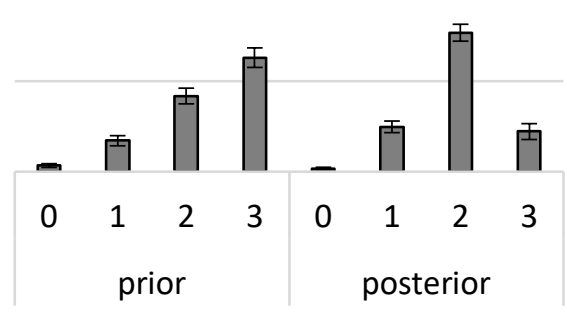

$\square$ some access 3

Figure 3 - Results of experiment 1, which attempted to replicate the study by (Goodman, Stuhlmüller, 2013). The graphs present the mean participant bet on each world state, varying the word the speaker used and the speaker's perceptual access. Error bars represent the standard error of the mean. 
Analogously to the original experiment, there was no effect of scenario and we collapsed data across this factor in all analyses. For clarity and ease of comparison with the original experimental results, we present analogous calculations, t-tests with additional Cohen's d effect sizes ( since effect size measures are taken to be more independent of sample size than p-values cf. ((Field 2013). We assumed that the threshold for significance adopted in the original paper was $\mathrm{p}=0.05$. Despite an explicit statement by the speaker in the scenario as to her state of knowledge of the context, some participants incorrectly judged the speaker as likely to have full access to the properties of all objects in the partial-access conditions (the bet that the speaker had complete knowledge when access $=1$ was $\mathrm{M}=36.66, \mathrm{SD}=37.25$, when access = $2, \mathrm{M}=48.33, \mathrm{SD}=39.57$; when access $=3, \mathrm{M}=88.98, \mathrm{SD}=23.8 \mathrm{o}$.)

As we were interested in the effects of varying knowledgeability, just as in the original experiment, we excluded trials in which the knowledge judgment was 'less than 70 in the expected direction'(Goodman and Stuhlmüller 2013). This means that if the speaker said he had seen all the objects, we excluded participants who bet less than 70 on the answer 'yes' in the control question. By contrast, if the speaker said he had seen one or two out of the three objects, we excluded participants who bet less than 70 on the answer 'no' in the control question.

We rejected 28 trials in the 'some access 1' condition, 38 trials in the 'some access 2 ' condition and 7 trials in the 'some access 3 ' condition.

Figure 3 shows the mean of bets on each option, as the speaker's access varied from one to three objects. As predicted, there was an effect of the speaker's access on the listener's interpretation (one-way ANOVA with bets on 3 objects having the property as a dependent variable, $\mathrm{F}(2)=6.647, \eta 2=.098, \mathrm{p}=.002$ (while in the original experiment $\mathrm{p}<0.001$ ).

We next performed comparisons to check that an implicature was drawn (posterior bets on 3 were bigger than bets on 2) when the speaker had complete knowledge, but not when the speaker had partial knowledge. In the complete-access condition, bets on 3 were less than bets on 2 (paired, directional $t$ test, $t(53)=6.50, p<0.001$ ). The effect size was $d=.89$.

In the partial-access conditions, the implicature did not occur (in the original paper the terminology used was that it was 'not cancelled,' but there were no time measures and the mechanisms of the RSA model instead predicted that the implicature was not formed, so we decided not to use the 'cancellation' terminology): Bets on 2 did not exceed bets on 3 when the speaker had access to 1 object (paired, directional $t$ test, $t(32)=-0.777, p=.443(p=.780$ in the original study)) The effect size was $\mathrm{d}=.22$.

However, in contrast to the original study, when the speaker's access was 2 objects, the implicature did occur (paired, directional $\mathrm{t}$ test, $\mathrm{t}(22)=2.646, \mathrm{p}=.015(\mathrm{p}=.210$ in the original study)). The effect size was $\mathrm{d}=.98$. For this reason, we do not fully replicate the original results, though this does not mean that there is no partial replication cf. (Gelman and Stern, 2006 ; Simonsohn, 2015). The null hypothesis adopted by the Authors of the original paper was that there was no implicature, while the tested hypothesis was that there was an implicature. Since the p value in the original study was way above the threshold of 0.05 , the Authors concluded that there was not enough evidence to reject the null hypothesis that there was no implicature. By contrast, our results show a p value below 0.05 , which provides us with grounds to reject the null hypothesis and conclude that there was an implicature in this condition (or at least that the implicature inference is stronger than in the results collected by goodman \& Stuhlmüller) ${ }^{7}$. Moreover, the effect size of .98 that we measured is a large, non-negligible effect size that supports our conclusion. Note that our elicitation of prior probability distribution (the 'almost always' statement) in the critical 'some access two' condition was as predicted. The prior bets on three were bigger than the prior bets on two and on one. The mean bets on zero objects that had the property were $=3 \cdot 3$ o, on one $=11.96$, on two $=39.74$ and on three $=45.00$.

If we only look at bets on 3 (see below), we see significantly lower bets in the complete-access condition than in the access 1 condition (unpaired, directional t test, $\mathrm{t}(85)=3.606, \mathrm{p}<.001$ ). The effect size was $\mathrm{d}=.88$.

\footnotetext{
${ }^{7}$ We thank a reviewer for pointing that the scalar implicature model from the study we are replicating is a quantitative model that does not predict categorical *implicature* vs. *no implicature* but rather graded, quantitative degrees of implicature (technically, just degrees of belief in different states), so the fact that there is a weak implicature rather than no implicature might suggest the model was miscalibrated (for example the speaker optimality parameter alpha might have been off, or a better linking function should have been used for the betting measure, see. (Franke et al. 2016)) or it could suggest the domain restriction idea described in the paper (a quantifier term ambiguity).
} 
Equally, when the speaker's access was 2 objects, the bets on three were significantly lower (unpaired, directional $t$ test, $\mathrm{t}(75)=0.997, \mathrm{p}=.322$ (while in the original study $\mathrm{p}<.001)$ ), $\mathrm{d}=.25$.

While there was no significant implicature in the 'some access 1' partial-access condition, there was a much greater tendency toward an implicature in the access 2 condition than in the access 1 condition (two-way ANOVA with bet as the dependent variable, and access ( 1 or 2$)$ and state ( 2 or 3 ) as independent variables, $F(2)=96.196, p<.001)$.

To sum up, the results obtained in our study can be considered a replication of the original experiment only in the case of the total-access condition and one partial-access condition, namely the 'some access one' condition. The results in the second partial-access condition, namely the 'some access two' condition revealed a much stronger implicature than in the original experiment.

\subsection{Discussion}

Our results suggest that a quantifier term ambiguity occurred during the experiment (see Figure 6). To provide experimental support for this hypothesis, we decided to conduct a study of scalar terms in Polish and observe whether the exact same issue as in the English results occurs in the Polish data. If the trend is cross linguistic, then this gives prima facie support for a possible ambiguity of the quantifier term in the experimental design.

\section{Experiment 2 - replication in the Polish language (scenario employing the term 'some' - 'niektóre')}

We conducted a replication of the original experiment in the Polish language. Below we present the results for conditions with the use of the quantifier term 'some,' which in Polish is 'niektóre.' We did this for two reasons: First, as presented above, there are some differences in the semantics of 'niektóre' in comparison to the English 'some,' which we suspected to be critical for replication. Second, we hypothesize that there might be a quantifier term ambiguity in the 'some access two' condition and it occurs regardless of the language employed for the experiment.

\subsection{Participants}

Just as in the original experiment, 5o participants were recruited through a Polish crowd-sourcing service, 'Research Online, ${ }^{8}$. We filtered out participants that were non-native speakers of the Polish language. All participants were students (female: $58 \%$; mean age: 26.6 years, $\mathrm{SD}=3.7$, age range: 25 -35 years). Since this is a per trial statistic (just as in the original experiment) each participant had three trials (150 trials collected in total) and we excluded trials for which participants failed a comprehension question, leaving a sample of go trials.

\footnotetext{
${ }^{8}$ Link to the platform: http://researchonline.pl
} 
We employed the exact same method and scenarios as in the original experiment, with two initial questions checking the participant's attention (described in detail in section 2.1). We translated the six scenarios to the Polish language (cf. Appendix). 
80

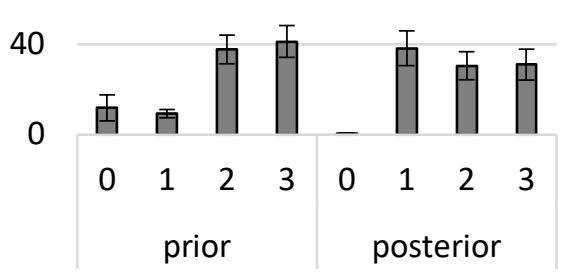

$\square$ some access 1

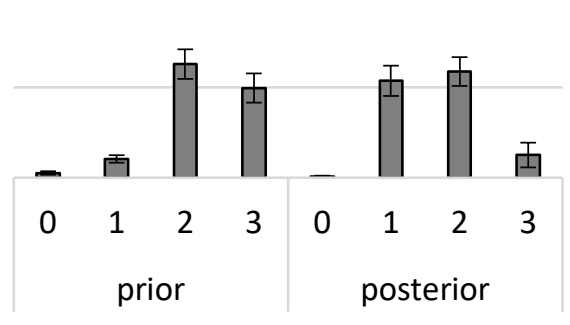

$\square$ some access 2

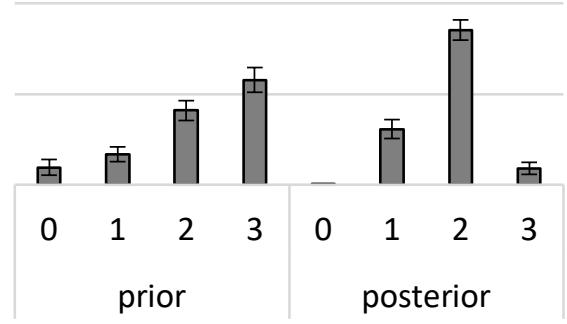

$\square$ some access 3

Figure 4 Replication in Polish with the participation of 5 o students, tested scenarios using 'some.' Error bars represent the standard error of the mean. 
Just as in the original study, there was no effect of scenario, so we collapsed data across this factor in all analyses. The speaker's access statement (e.g., "I have looked at 2 of the 3 letters") was not a perfect manipulation of knowledgeability: in the partial-access conditions, some participants judged that the speaker was likely to know exactly how many objects had the property. In other words, in the control question in the partial-access conditions ('do you think the speaker knows exactly how many of the objects had the property') some participants bet less than 70 on the answer 'no.' (The bet that the speaker had complete knowledge when access $=1$ was $\mathrm{M}=39.18, \mathrm{SD}=41.56$; when access $=2, \mathrm{M}=46.68, \mathrm{SD}$ $=42.85$; when access $=3, \mathrm{M}=89.24, \mathrm{SD}=23.58$.)

\begin{tabular}{ccc}
\hline & Original & Replication \\
Access 1 & 27.1 & 39.18 \\
& $(4.9)$ & $(41.56)$ \\
Access 2 & 34.8 & 46.68 \\
& $(.7)$ & $(42.85)$ \\
Access 3 & 93.0 & 89.24 \\
& $(2.7)$ & $(23.58)$ \\
\hline
\end{tabular}

Table 1 Means of bets that the speaker has complete knowledge in the partial-access (Access 1, Access 2) and complete-access (Access 3 ) conditions as well as the standard deviations, data from experiment 2.

Analogously to the original experiment, we were interested in the effects of varying knowledgeability, we therefore excluded trials in which the knowledge judgment was less than 70 in the expected direction. We rejected 24 participants in the 'some access 1' condition, 28 participants in the 'some access 2' condition and 8 participants in the 'some access 3' condition.

Figure 4 shows the mean of bets on each option as access varied. As predicted, there was an effect of the speaker's access on the listener's interpretation (one-way ANOVA with bets on 3 as the dependent variable, $F(2)=7.54, \eta 2=.148$, $\mathrm{p}<.001)$.

We then performed comparisons to check that an implicature was drawn when the speaker had complete knowledge, but not when the speaker had partial knowledge. In the complete-access condition, bets on 3 were less than bets on 2 (paired, directional $\mathrm{t}$ test, $\mathrm{t}(41)=10.103, \mathrm{p}<.001$ ). The effect size was $\mathrm{d}=2.65$.

In the 'some access one' partial-access condition, there was no implicature. Bets on 2 did not exceed bets on 3 when the speaker had access to 1 object (paired, directional $\mathrm{t}$ test, $\mathrm{t}(25)=.048, \mathrm{p}=.962$ ). The effect size was $\mathrm{d}=.01$.

However, contrary to the original study, when access was 2, the implicature was present (or much stronger) (paired, directional $\mathrm{t}$ test, $\mathrm{t}(21)=3.769, \mathrm{p}=.001$ (compared to the original experiment, in which $\mathrm{p}=.210)$ ). The effect size was $\mathrm{d}=$ 1.33. For this reason we can consider our results to be only a partial replication of the original experiment. Nevertheless, this time the prior elicitation was not as predicted: mean participant prior bets on zero objects having the property were 2.09 , on one $=8.27$, on two $=5$ o.09, and on three $=39.55$.

If we look at only bets on 3 , we see significantly lower bets in the complete-access condition than the access 1 condition (unpaired, directional $\mathrm{t}$ test, $\mathrm{t}(33)=3.247, \mathrm{p}=.003$ ). The effect size was $\mathrm{d}=.94$; by contrast, no such trend was present in the access 2 condition (unpaired, directional $t$ test, $t(62)=.518, p=.6 \circ 6$ (contrary to the original experiment where $p$ $<.001)$ ) and the effect size measure indicates a small effect, $\mathrm{d}=.14$.

While there was no significant implicature in the 'some access 1' partial-access condition, there was a greater tendency toward implicature in the access 2 condition than the access 1 condition, as predicted by the model (two-way ANOVA with bet as the dependent variable and access (1 or 2$)$ and state (2 or 3 ) as independent variables, $\mathrm{F}(1)=33.434$, $\eta 2=.66, \mathrm{p}<.001)$. 
Both of the experiments testing the quantifier term 'some' interpretation that we carried out in the English and Polish languages proved to be only a partial replication of the original experiment. Also, contrary to our predictions, the replication failure occurred in the exact same 'some access two' condition in both languages; while in the original experiment the 'some access two' condition generated no implicature, in our experiments in both languages the implicature was present. The prior elicitation in the Polish version of the experiment in the critical condition was imperfect. Participants in their answers to the question on prior probability distribution may have neglected the information that the objects almost always had the property. This might have been because the phrase: 'letters to Laura's company almost always have cheques inside' was enriched to the unpredicted interpretation 'not always.' However, the English version generated answers on the prior probability distribution that conformed to the predictions and the very same problematic result in the question on the posterior probability distribution. If the same issue occurs in both languages, we suspect that this is due to an ambiguity of the quantifier term. We now turn to a detailed discussion of this hypothesis.

\section{Discussion - Experiment 1 and 2}

Our hypothesis presented in Figure 5 is that that the scalar terms used in both experiments in the 'some access two' condition were ambiguous in terms of their. This effect was observed in both the English and Polish versions of the experiment.

Incomplete knowledge ("I have looked at 2 of the 3 letters. Some of the letters have cheques inside.")

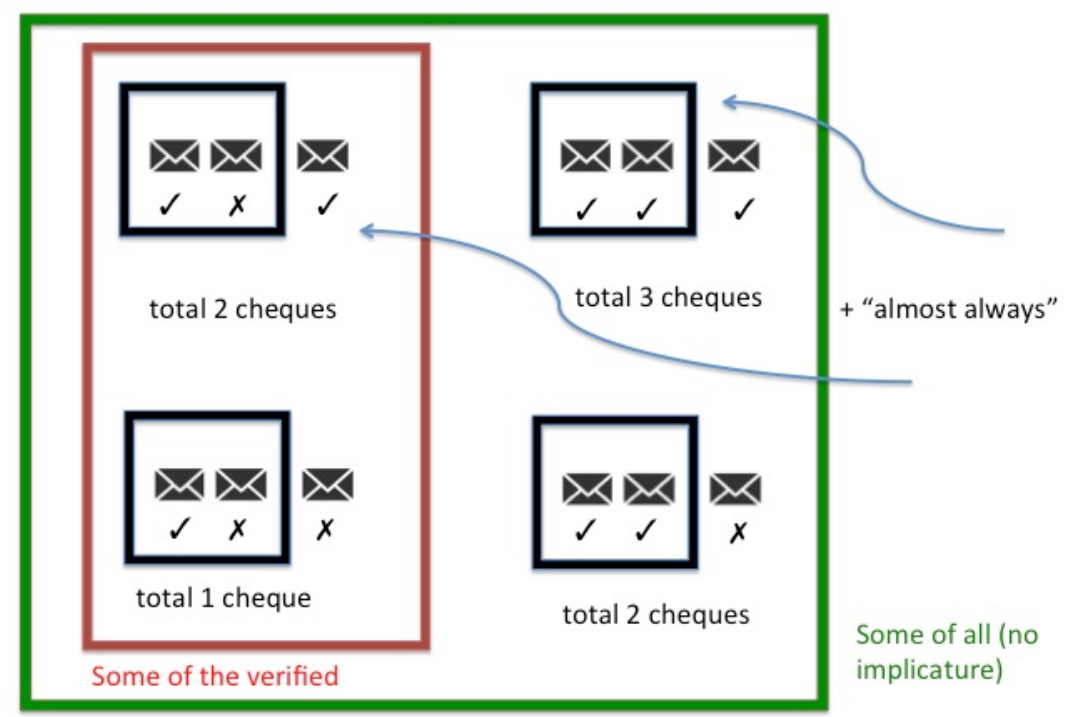

Figure 5 Quantifier term ambiguity in the 'Some access two' condition. The small black boxes present the letters whose content was verified by the speaker. 
We suspect that there was no replication in the 'some access two' condition because participants did not interpret the speaker's words as predicted. Suppose the speaker said:

(V.) I have looked at 2 out of the 3 letters. Some of the letters have cheques inside.

In this case, the prediction was that the sentence would be understood as 'some of all of the objects have the salient feature'; however, we suspect that participants instead adopted the interpretation that some of the verified objects (for instance letters) had the salient feature (for instance cheques inside).

Let us focus on the 'Some" access 2' condition presented in Figure 5. If you interpret (XV) as 'some of the verified letters have cheques inside' and you take this to mean not all of the verified letters have cheques inside, then you understand that only one of the two verified letters contains a cheque. Since there are three letters in total, the third letter that was not verified by the speaker could either contain a cheque or not. If it contains a cheque, then there is a total of two cheques in the three letters. If it does not contain a cheque, then the total of cheques in the three letters is one. Thus, the tendency is to bet higher sums on one or two letters containing cheques rather than all three letters containing cheques. Moreover, since the scenarios start with a statement that letters almost always have cheques inside and you consider two cases, namely that there are either one or two cheques in the total three letters, you will bet more on two letters having cheques inside than one letter having a cheque inside.

By contrast to the actual hypothesized interpretation that we suggest, the purpose of the experiment was to make participants interpret $(\mathrm{V})$ as 'some of all of the letters have cheques inside' and derive an uncertainty implicature. This is because such an interpretation enables additional situations in which either both of the verified letters have cheques inside and the third unverified letter does not, or both of the verified letters have cheques inside and the third unverified letter also does. Since the scenarios start with the information that letters almost always have cheques inside, the aim of the experiment was to guide participants to bet equally high on one, two and three of the letters having cheques inside and thus detect no implicature in the results. We suspect that since participants failed to grasp the intended interpretation, the implicature appeared.

To perform a quantitative check of our hypothesis we decided to take a closer look at the 'some access two' condition in the experiments we performed in both the English and Polish languages. We compare the difference in mean bets on one and two objects having the property (we call this A) and mean bets on two and three objects having the property (we call this B). If the differences in means are approximately equal or A is bigger than $\mathrm{B}$, then this would give prima facie evidence that the interpretation the Authors of the original experiment intended is being adopted by participants. This is because if the difference between bets on two and three is smaller than the difference between bets on one and two, then participants make high bets on three (all) of the objects having the property in question and the implicature does not arise. By contrast, if $\mathrm{A}$ is smaller than $\mathrm{B}$, then this gives prima facie evidence that the unintended interpretation, namely 'some of the verified letters', have been adopted by participants. This is because if the difference between bets on one and two is smaller than the difference between bets on two and three, then participants bet less on three (all) of the objects having the property in question and an implicature appears.

In the experiment we carried out in English, in the 'some access two' condition the difference in the means between bets on 'one' and 'two' objects having the property (called A) is 21.56 points, paired $t(22)=-2.8, p=.011$ and its effect size (Cohen's d) is .95. The difference in the means between bets on 'two' and 'three' objects having the property (called B) is 24.14, paired $t(22)=2.646, \mathrm{p}=.015$ and its effect size (Cohen's $\mathrm{d}$ ) is .98. If we assume that $\mathrm{A}<\mathrm{B}$ is 'some of the 
checked,' $\mathrm{A}>\mathrm{B}$ 'some of all' and 'A $\approx \mathrm{B}$ ' 'some of all,' then we get $\mathrm{A}<\mathrm{B}$ 'some of the checked,' which hints not only at a possible ambiguity of the quantifier term in the experimental design, but also that the participants adopted a different interpretation of the utterance than the one predicted.

Analogously, in the experiment we did in the Polish language, in the 'some access two' condition, the difference in the means between bets on 'one' and 'two' objects having the property (called A) is 4.09 points, paired $t(42)=0.44, \mathrm{p}$ $=.330$ and its effect size (Cohen's d) is .13. The difference in the means between bets on 'two' and 'three' objects having the property (called $B$ ) is 36.82 and its effect size (Cohen's d) is 1.33, t $(42)=4,40, p<$.ool. If we assume that $A<B$ is 'some of the verified,' $\mathrm{A}>\mathrm{B}$ 'some of all' and 'A $\approx \mathrm{B}$ ' 'some of all,' then we get $\mathrm{A}<\mathrm{B}$ 'some of the verified,' which again suggests a possible ambiguity in the experimental design.

Note that this ambiguity cannot arise in the remaining partial-access condition, namely 'some access one,' because if only one verified letter contains a cheque, then the remaining two letters can both contain a cheque. Thus participants would in these cases consider the possibility that all three letters have cheques inside and make appropriate bets on 3 objects having the property.

To address these issues and investigate further the quantifier term ambiguity we performed an experiment in Polish with a quantifier term disambiguation using the same experimental design as in previous experiments but the results were inconclusive: for a detailed description see Appendix. For this reason, we redesign a new experiment. We use a between-subjects design in order to avoid a per trial statistic which leads to only some of the trials from the same participants being used. We increase the number of objects at stake to five so as to mitigate the artificiality of claiming that "some of the objects have the property" when only one out of three objects has the property as it is then more natural to employ the numeral "one". Finally, to test whether the quantifier term ambiguity hypothesis is correct, we employ three different formulations in experiment 3 . To check whether participants beliefs about what is communicated and beliefs about the world diverge, we add an additional question on communicated content.

\section{$5 \quad$ Experiment 3}

\subsection{Participants}

984 participants were recruited online via Amazon Mechanical Turk to perform a preregistered survey ${ }^{9}$. Since the present experiment is between subjects, we calculated the number of participants on the basis of a predictive power analysis carried out with the Gpower software. The IP address location was restricted to the USA. Participants who failed the attention check, took less than a minute for the entire survey or failed a comprehension question were excluded, leaving a sample of 665 participants (female: $49 \%$; mean age: 39.4 years, $\mathrm{SD}=12.2$, age range: $20-82$ years).

\subsection{Methods and materials}

For experiment 3 we use a between subjects' design: five number terms (one v. two v. three $v$. four v. five) $\mathrm{x}$ three formulations (some of the phones v. some of the phones that I have looked at v. some of the phones that I must repair). Having

\footnotetext{
${ }^{9}$ Link to preregistration: https://aspredicted.org/blind.php? $\mathrm{x}=\mathrm{pc} 4 \mathrm{n} 5 \mathrm{~m}$
} 
passed an attention check, participants were randomly presented with the following vignette (labels in bold were removed):

Broken Sigo phones almost always have burned-out transistors. Ben must repair five broken Sigo phones.

Q1 EX ANTE: How many of the five phones do you think have burned-out transistors?

Ben tells you on the phone: "I have looked at one/two/three/four/five of the five phones. Some of the phones/some of the phones that I have looked at/some of the phones that I must repair have burned-out transistors."

Q2 EX POST: Now how many of the five phones do you think have burned-out transistors?

/order of questions below randomized:/

Q3 KNOWLEDGE: Do you think Ben knows exactly how many of the five phones have burned-out transistors? (yes/no)

Q4 MEANING: What did Ben want to communicate with the utterance "Some of the phones that I have looked at/that I must repair have burned-out transistors."?

a) Only some but not all of the phones have burned-out transistors.

b) At least some and maybe all of the phones have burned-out transistors.

\section{$5 \cdot 3 \quad$ Results}

The speaker's access statement (e.g., "I have looked at 2 of the 5 phones") was a successful manipulation of knowledgeability: in the partial-access conditions, most participants judged that the speaker did not know exactly how many objects had the property, while in the complete access condition ("I have looked at 5 of the 5 phones") most participants judged that the speaker knew how many objects had the property (cf. Figure 6). 


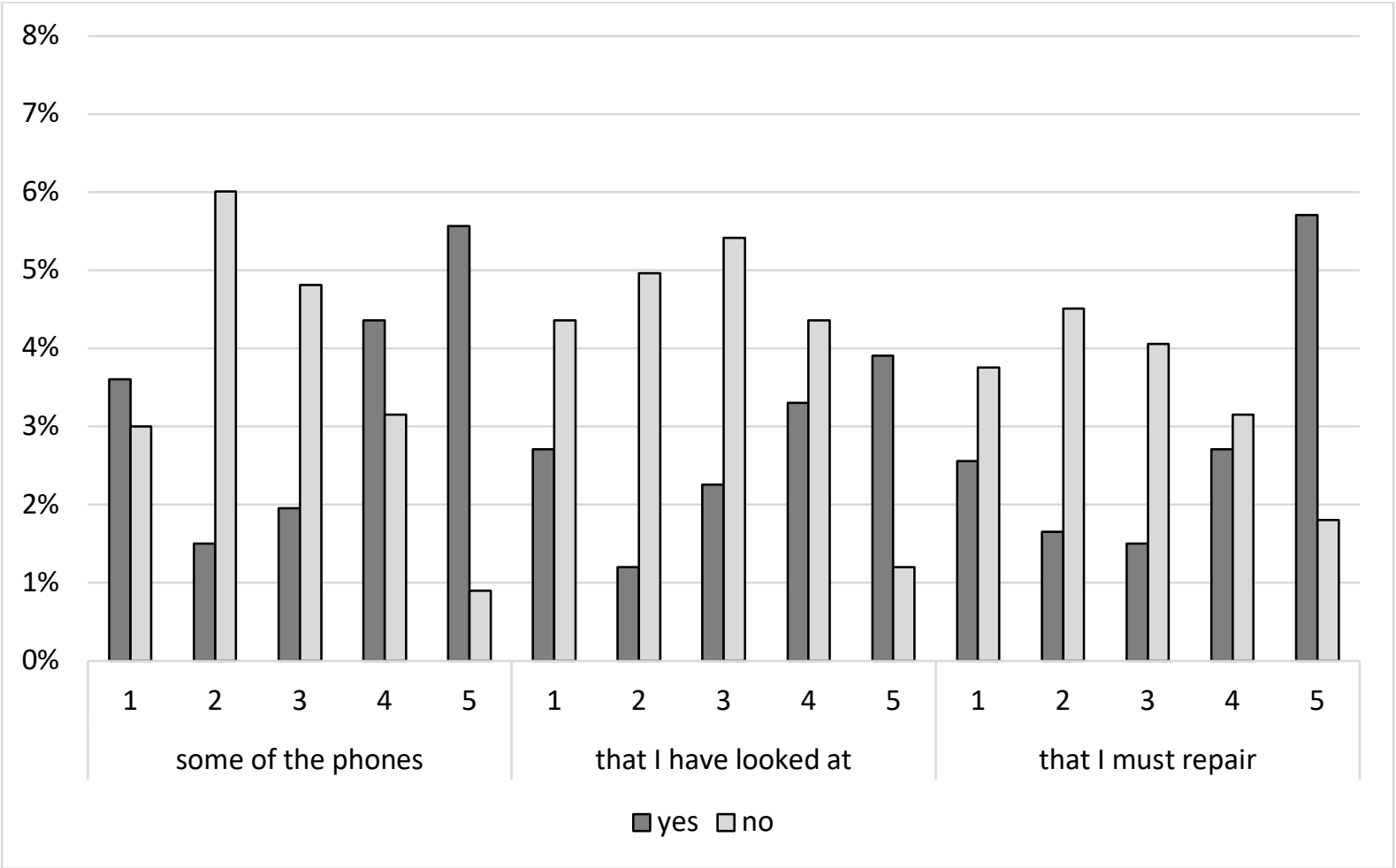

Figure 6 Percentage proportion of participants per condition that judged that speaker had knowledge of how many objects have the property ("yes" answers) or did not have knowledge of how many objects have the property ("no" answers). The numbers on the $\mathrm{x}$-axis represent the levels of perceptual access.

The answers on the prior probability distribution question were as predicted, cf. Figures 7,8 and 9 . We next performed comparisons to check that an implicature was drawn when the speaker had complete knowledge, but not when the speaker had partial knowledge. In the complete-access condition, bets on 5 were less than bets on 4 in all three formulations (paired, directional t test, $\mathrm{t}(126)=7.368, \mathrm{p}<.001$ ). The effect size was $\mathrm{d}=.99$. For all formulations separately, all $p s$ were equal to or below .oo1 (cf. Table 2).

In the partial-access conditions, the implicature did appear when access was three and four for all formulations: all $p s$ below .049 in a paired, directional t test comparing bets on four and five objects having the property (cf. Table 2).

When access was "one", the implicature was cancelled in all formulations: a paired, directional t test comparing bets on four and five objects having the property revealed that all $p s$ were above .o71 (cf. Table 2).

Interestingly, when access was "two", the implicature appeared in the formulation "some of the phones" (paired, directional $\mathrm{t}$ test comparing bets on four and five objects having the property: $\mathrm{t}(49)=3.875, \mathrm{p}<.001, \mathrm{~d}=.86)$. By contrast, when access was "two" and the formulation was "some of the phones that I must repair" there was no implicature (paired, directional $\mathrm{t}$ test comparing bets on four and five objects having the property: $\mathrm{t}(4 \mathrm{O})=.572, \mathrm{p}=.57 \mathrm{O}, \mathrm{d}=.15)$. However, when access was "two" and the formulation was "some of the phones that I have looked at", the implicature was merely borderline significant (paired, directional $t$ test comparing bets on four and five objects having the property: $\mathrm{t}(40)=1.976$, $\mathrm{p}=.055, \mathrm{~d}=.46$ ). For all t-tests cf. Table 2 as well as Figures $7-11$. 


\begin{tabular}{|c|c|c|c|c|c|c|c|c|c|c|c|c|}
\hline \multirow[t]{3}{*}{$\underline{\text { Access }}$} & \multicolumn{12}{|c|}{ Formulations } \\
\hline & \multicolumn{4}{|c|}{ Some of the phones } & \multicolumn{4}{|c|}{ That I have looked at } & \multicolumn{4}{|c|}{$\underline{\text { That I must repair }}$} \\
\hline & $t(d f)$ & $p$ & Cohen's d & $95 \% C I$ & $t(d f)$ & $p$ & Cohen's d & $95 \% C I$ & $t(d f)$ & $p$ & Cohen's d & $95 \% C I$ \\
\hline 1 & $.79(43)$ & $.43^{2}$ & .19 & {$[-8.66 ; 19.89]$} & $-1.04(46)$ & .303 & .23 & {$[-20.29 ; 6.46]$} & $1.86(41)$ & .071 & .48 & {$[-1.39 ; 32.67]$} \\
\hline 2 & $3.88(49)$ & $<.001$ & .86 & [11.73;36.99] & $1.98(40)$ & .055 & .46 & {$[-\cdot 31 ; 27 \cdot 73]$} & $.57(40)$ & .570 & .15 & {$[-13 \cdot 15 ; 23 \cdot 54]$} \\
\hline 3 & $2.04(44)$ & .048 & .43 & {$[.12 ; 21.75]$} & $4.5^{2}\left(5^{\circ}\right)$ & $<.001$ & .92 & [12.99;33.76] & $2.85(36)$ & .007 & .62 & {$[4.11 ; 24.53]$} \\
\hline 4 & $3 \cdot 54(49)$ & .001 & .73 & {$[8.16 ; 29.52]$} & $3 \cdot 30\left(5^{\circ}\right)$ & .002 & .65 & {$[6.15 ; 25.22]$} & $3.78(38)$ & .001 & .90 & [11.96;39.58] \\
\hline 5 & $3.62(42)$ & .001 & .86 & {$[9.92 ; 34.92]$} & $3.99(33)$ & $<.001$ & 1.03 & {$[12.67 ; 38.86]$} & $5.07(49)$ & $<.001$ & 1.08 & [16.43;38.01] \\
\hline
\end{tabular}

Table 2 Paired, directional t tests comparing ex post bets on four and five objects having the property for all conditions: three formulations times five access options. 
80

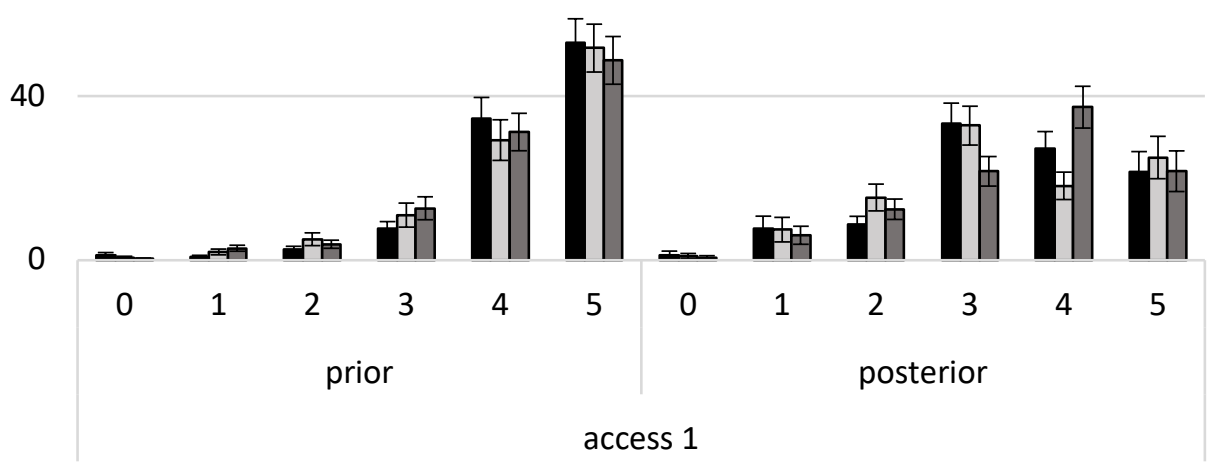

some of the phones $\square$ that I have looked at $\quad \square$ that I must repair

Figure 7 Mean bets on each of the five objects having the property for access equal to one and for the three formulations ("some of the phones" vs "some of the phones that I have looked at" vs. "some of the phones that I must repair"). Error bars depict the standard error of the mean.

80

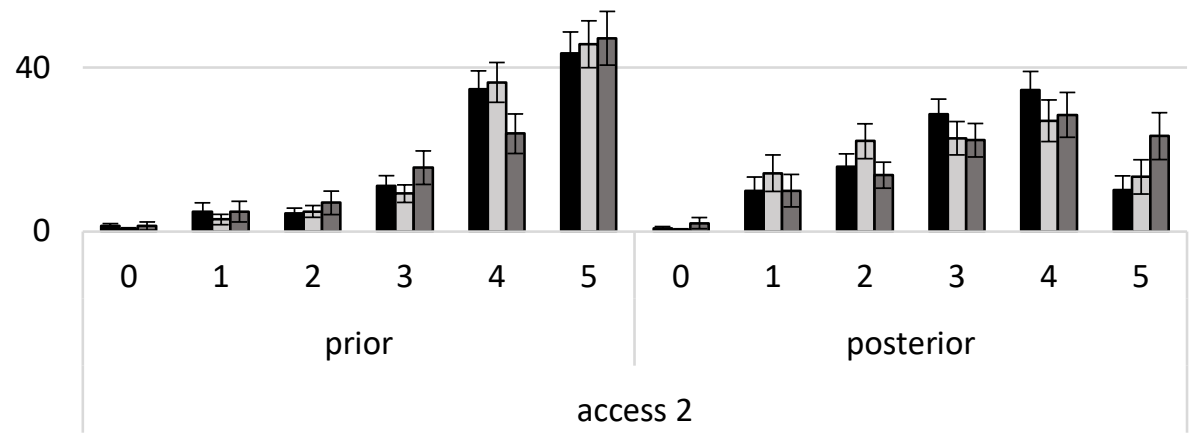

some of the phones $\quad \square$ that I have looked at $\quad \square$ that I must repair

Figure 8 Mean bets on each of the five objects having the property for access equal to two and for the three formulations ("some of the phones" vs "some of the phones that I have looked at" vs. "some of the phones that I must repair"). Error bars depict the standard error of the mean. 
80

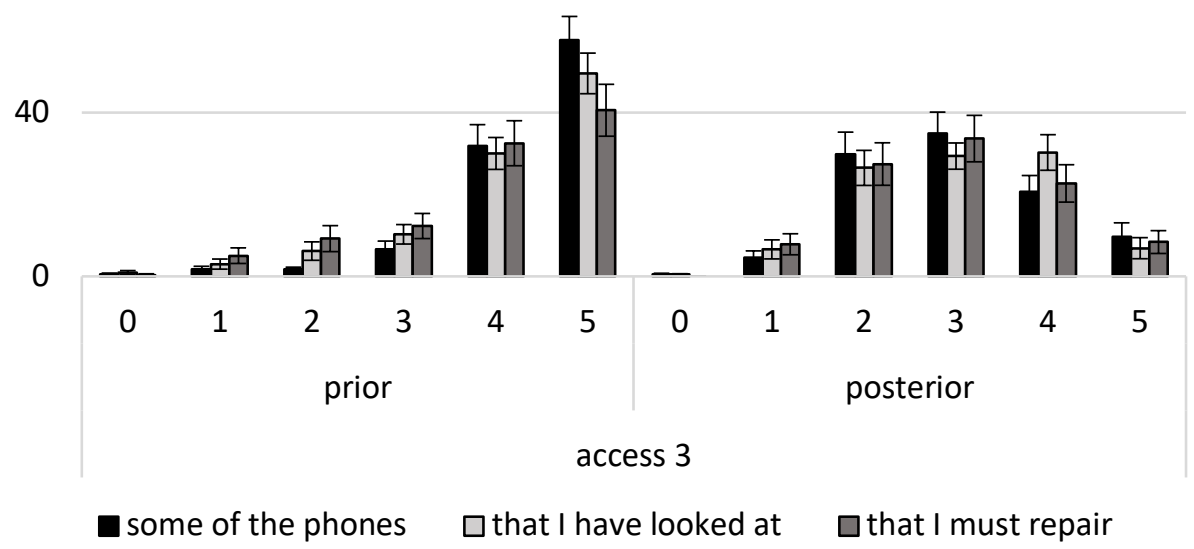

Figure 9 Mean bets on each of the five objects having the property for access equal to three and for the three formulations ("some of the phones" vs "some of the phones that I have looked at" vs. "some of the phones that I must repair"). Error bars depict the standard error of the mean.

80

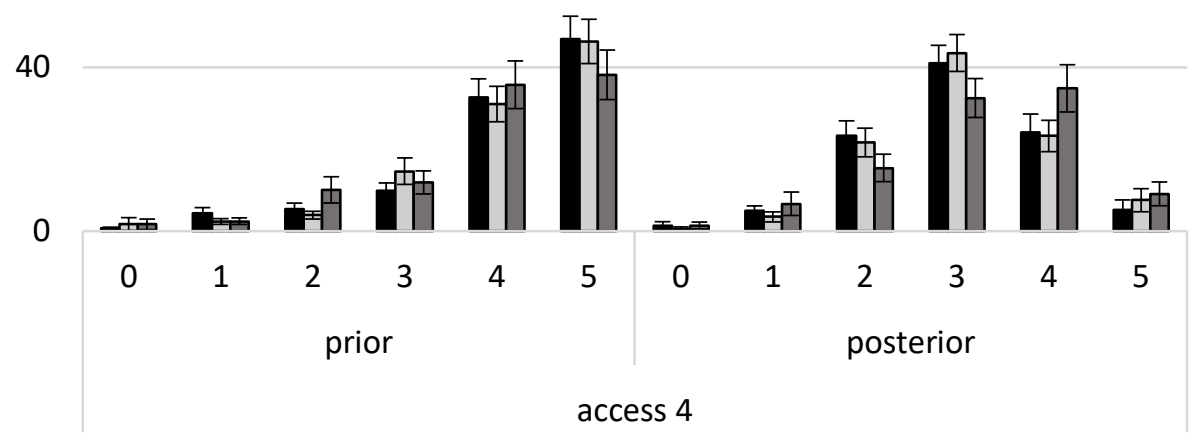

some of the phones $\square$ that I have looked at $\quad \square$ that I must repair

Figure 1o Mean bets on each of the five objects having the property for access equal to four and for the three formulations ("some of the phones" vs "some of the phones that I have looked at" vs. "some of the phones that I must repair"). Error bars depict the standard error of the mean. 


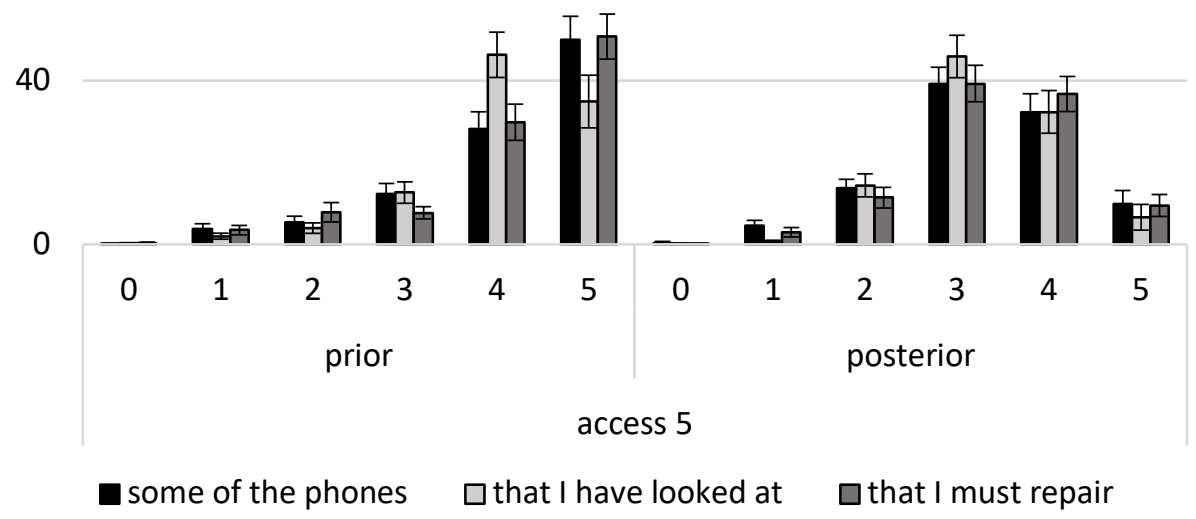

Figure 11 Mean bets on each of the five objects having the property for access equal to five and for the three formulations ("some of the phones" vs "some of the phones that I have looked at" vs. "some of the phones that I must repair"). Error bars depict the standard error of the mean.

Most participants judged that, independently of the formulation, in the partial access conditions, the speaker communicated that "some but not all of the objects" had the property (cf. Figure 12).

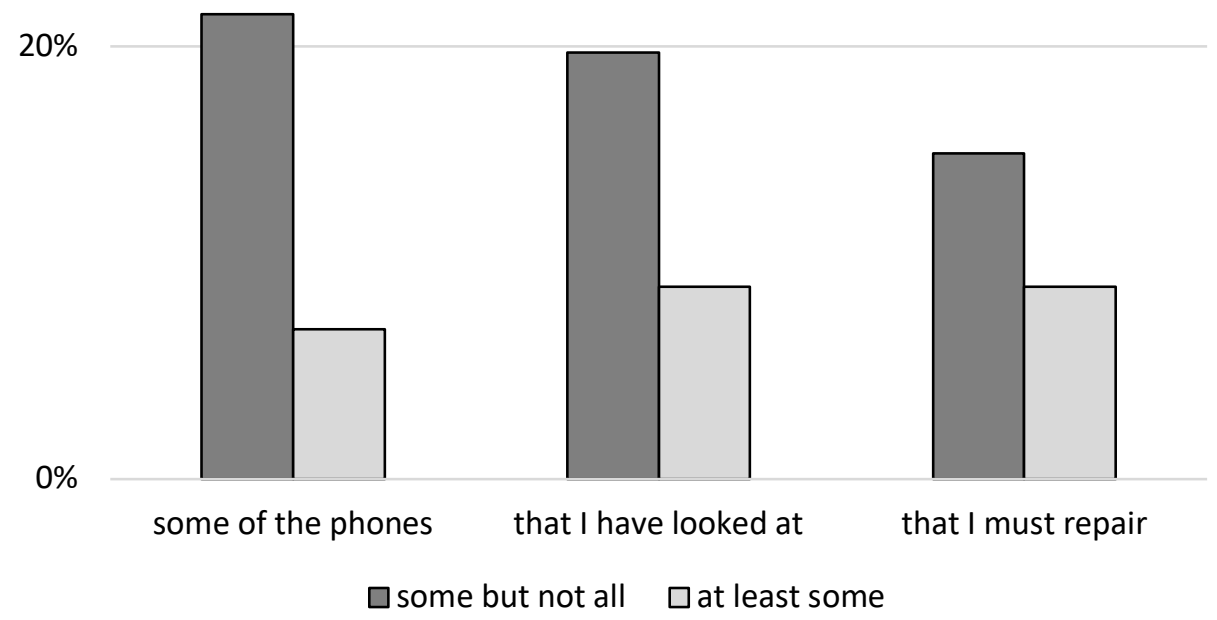

Figure 12 Proportion of participants that judged that the speaker communicated that either "some but not all" or "at least some" of the objects have the property for each of the three tested formulations. We excluded the complete access formulation (I have looked at 5 of the 5 phones). 
As predicted, in the question on ex ante probability distribution the 'almost always' formulation is driving the assessment of ex ante probability to be highest on 4 or 5 objects out of 5 to have the relevant property in question. The 'some that I must repair' formulation combined with the answer on the question on ex ante probability as well as the level of uncertainty is driving the assessment of ex post probability to be highest on either 3,4 or 5 objects having the relevant property in question. The assessment of the communicated content through the utterance using the term 'some' is different than the assessment of the ex post probability distribution on objects having the relevant property: in all partial access conditions, most participants judged that the speaker communicated that "some but not all" of the objects have the relevant property. Thus, in uncertainty contexts beliefs about what is communicated and beliefs about the state of the world diverge: the RSA gives robust predictions about reasoning concerning the state of the world.

With the formulation "that I have looked at", as predicted, there was a greater tendency to lower the bets on 3,4 and 5 objects having the property than with the alternative formulation ("that I must repair"): in the access two condition, the implicature was merely borderline significant with a much reduced effect size compared to the other two formulations. Thus, the results suggest that the quantifier term ambiguity is present. They also suggest that the interaction between prior and posterior probability distribution is conform to the predictions of the RSA model: the previous failures in replication were most probably due to the ambiguity of the quantifier. Finally, we should note that the ex post bets on 5 were low in all conditions, and thus a tendency to infer the implicature was present. Thus, even in uncertainty contexts, participant tend to make the inference 'not all' out of 'some'.

\section{General discussion}

The performed experiments support the hypothesis that there is a systematic and cross-linguistic interaction between utterance understanding and world knowledge shared by interlocutors. However, our results provide important insights into two important issues of the RSA model and experimental design: the priors issue and the quantifier term ambiguity issue.

As far as the priors issue is concerned, out of the two experiments we carried out in the Polish language with the quantifier term 'some' (experiments 2 and 3), only experiment 2 provided us with data that depicted a prior elicitation that was contrary to predictions, while both experiments provided us with data that depicted posterior elicitation contrary to theoretical predictions in the 'some access two' condition. In other words, regardless of whether participants ignored or considered the information that the objects almost always had the relevant property (and employed a prior probability distribution based on their everyday experience), they inferred the scalar implicature, contrary to the predictions of the model in the 'some access two' partial-access condition. Consequently, we need a different explanation of our only partial replication of the study that tested the interpretation of the quantifier term 'some.' Moreover, we found analogous differences in the data concerning the quantifier term 'some' from experiment 1 in the English language and in experiment 2 in the Polish language. While the incorrect prior elicitation was most probably due to the experiments being underpowered, the ex post probability distributions remained mysterious. This lead us to our second issue, namely quantifier term ambiguity.

The second issue is a suspected quantifier term ambiguity due to the experimental design in the 'some access two' condition, which leads to lack of replication of results in this condition in both tested languages. In experiment 3 for the 
formulation "some of the checked", there was a greater tendency to lower the bets on 3,4 and 5 objects having the property than with the alternative formulation ("some of all"): in the access two condition, the implicature was merely borderline significant with a much reduced effect size compared to the other two formulations. However, the implicature inference appeared in all the remaining partial access conditions.

\section{$7 \quad$ Conclusion}

Our overall results suggest that the quantifier term in the original vignettes is ambiguous, while the RSA gives correct predictions of a disambiguated quantifier term employment, rather than an ambiguous one.

It remains a project for future studies to formalize the contextual mechanism presented in experiment 3 and create an updated version of the RSA model cf. (Degen et al. n.d.) (Qing et al. 2016) (Tessler et al. 2017)

\section{$8 \quad$ References}

Atlas, J. D., \& Levinson, S. C. (1981). It-Clefts, informativeness and logical form: Radical Pragmatis (Revised Standard Version). In P. Cole (Ed.), Radical pragmatics. New York: Academic Press.

Bergen, L., \& Grodner, D. J. (2012). Speaker knowledge influences the comprehension of pragmatic inferences. Journal of Experimental Psychology: Learning, Memory, and Cognition, 38(5), 1450-146o. https://doi.org/10.1037/aoo2785o

Bergen, L., Levy, R., \& Goodman, N. (2016). Pragmatic reasoning through semantic inference. Semantics and Pragmatics, 9. https://doi.org/10.3765/sp.9.20

Bott, L., \& Noveck, I. A. (2004). Some utterances are underinformative: The onset and time course of scalar inferences. Journal of Memory and Language, 51(3), 437-457. https://doi.org/10.1016/j.jml.2004.05.006

Chierchia, Genaro, Spector, B., \& Fox, D. (2011). The grammatical view of scalar implicatures and the relationship between semantics and pragmatics. In P. Portner, C. Maienborn, \& K. von Heusinger (Eds.), Handbook on semantics. Berlin: De Gruyter.

Chierchia, Gennaro. (2006). Broaden Your Views: Implicatures of Domain Widening and the "Logicality" of Language. Linguistic Inquiry, 37(4), 535-59o. https://doi.org/10.1162/ling.2006.37.4.535

de Carvalho, A., Reboul, A. C., Van der Henst, J.-B., Cheylus, A., \& Nazir, T. (2016). Scalar Implicatures: The Psychological Reality of Scales. Frontiers in Psychology, 7. https://doi.org/10.3389/fpsyg.2016.01500

Degen, J., Tessler, M. H., \& Goodman, N. D. (n.d.). Wonky worlds: Listeners revise common ground when utterances are odd.

Field, A. P. (2013). Discovering statistics using IBM SPSS statistics: and sex and drugs and rock " $n$ " roll (4th edition.). Los Angeles: Sage. 
Fodor, J. A. (1983). The modularity of mind: an essay on faculty psychology. Cambridge, Mass: MIT Press.

Fox, D. (2007). Free Choice and the Theory of Scalar Implicatures. In U. Sauerland \& P. Stateva (Eds.), Presupposition and Implicature in Compositional Semantics (pp. 71-120). London: Palgrave Macmillan UK. https://doi.org/10.1057/9780230210752_4

Frank, M. C., \& Goodman, N. D. (2012). Predicting Pragmatic Reasoning in Language Games. Science, 336(6o84), 998-998. https://doi.org/10.1126/science.1218633

Franke, M., Dablander, F., Scholler, A., Bennett, E., Degen, J., Tessler, M. H., et al. (2016). What does the crowd believe? A hierarchical approach to estimating subjective beliefs from empirical data. https://cogsci.mindmodeling.org/2016/papers/o46o/index.html

Gelman, A., \& Stern, H. (2006). The Difference Between "Significant" and "Not Significant" is not Itself Statistically Significant. The American Statistician, 6o(4), 328-331. https://doi.org/10.1198/ooo3130o6X152649

Geurts, B. (2010). Quantity implicatures. Cambridge ; New York: Cambridge University Press.

Goodman, N. D., \& Frank, M. C. (2016). Pragmatic Language Interpretation as Probabilistic Inference. Trends in Cognitive Sciences, 20(11), 818-829. https://doi.org/10.1016/j.tics.2016.08.005

Goodman, N. D., \& Stuhlmüller, A. (2013). Knowledge and Implicature: Modeling Language Understanding as Social Cognition. Topics in Cognitive Science, 5(1), 173-184. https://doi.org/10.1111/tops.12007

Hawkins, X. D., \& Goodman, N. D. (forthcoming). Questions and answers in dialogue.

Honselaar, Wi. (1983). Universal quantifiers in Russian. Studies in Slavic and General Linguistics, 3, $213-246$.

Horn, L. R. (1972). On the semantic properties of logical operators in English. Los Angeles, CA: University of California doctoral dissertation.

Horn, L. R. (2006). Implicature. In The handbook of pragmatics. Malden, MA; Oxford: Blackwell. http://dx.doi.org/10.1002/9780470756959. Accessed 2 May 2015

Montague, R. (1973). The proper treatment of quantification in ordinary English. In P. Suppes, J. Moravcsik, \& J. Hintikka (Eds.), Approaches to Natural Language (pp. 221-242). Dodrecht.

Noveck, I. A. (2001). When children are more logical than adults: experimental investigations of scalar implicature. Cognition, 78(2), 165-188. https://doi.org/10.1016/Soo1o-0277(oo)oo114-1

Noveck, I. A., \& Posada, A. (2003). Characterizing the time course of an implicature: An evoked potentials study. Brain and Language, 85(2), 203-210. https://doi.org/10.1016/Soo93934X(०3)ooo53-1

Noveck, I. A., \& Sperber, D. (2004). Experimental pragmatics. Basingstoke, Hampshire; New York: Palgrave Macmillan. http://site.ebrary.com/id/10262251. Accessed 23 November 2015

Papafragou, A., \& Musolino, J. (2003). Scalar implicatures: experiments at the semantics-pragmatics interface. Cognition, 86(3), 253-282. https://doi.org/10.1016/Soo1o-o277(o2)oo179-8 
Papafragou, A., \& Tantalou, N. (2004). Children's Computation of Implicatures. Language Acquisition, 12(1), 71-82. https://doi.org/10.1207/s15327817la1201_3

Piasecki, M. (2002).Język modelowania znaczenia polskiej frazy nominalnej [The language of modeling the meaning of the Polish nominal phrase]. Wrocław: Doctoral thesis.

Pouscoulous, N., Noveck, I. A., Politzer, G., \& Bastide, A. (2007). A Developmental Investigation of Processing Costs in Implicature Production. Language Acquisition, 14(4), 347-375. https://doi.org/10.108o/10489220701600457

Qing, C., Goodman, N. D., \& Lassiter, D. (2016). A rational speech-act model of projective content. https://cocolab.stanford.edu/papers/QingGoodmanLassiter2o16-Cogsci.pdf

Russell, B. (2006). Against Grammatical Computation of Scalar Implicatures. Journal of Semantics, 23(4), 361-382. https://doi.org/10.1093/jos/ffloo8

Sauerland, U. (2004). Scalar Implicatures in Complex Sentences. Linguistics and Philosophy, 27(3), 367-391. https://doi.org/10.1023/B:LING.ooooo23378.71748.db

Sauerland, U. (2012). The Computation of Scalar Implicatures: Pragmatic, Lexical or Grammatical?: Computation of Scalar Implicatures. Language and Linguistics Compass, 6(1), 36-49. https://doi.org/10.1002/lnc3.321

Simonsohn, U. (2015). Small Telescopes: Detectability and the Evaluation of Replication Results. Psychological Science, 26(5), 559-569. https://doi.org/10.1177/o956797614567341

Spector, B. (2003). Scalar implicatures: exhaustivity and Gricean reasoning. In B. ten Caten (Ed.), Proceedings of the Eighth ESSLLI Student Session (pp. 277-88). Vienna, FOLLI.

Sperber, D., \& Wilson, D. (2002). Pragmatics, Modularity and Mind-reading. Mind and Language, $17(1$ \& 2), 3-23. https://doi.org/10.1111/1468-0017.00186

Sperber, D., \& Wilson, D. (2006). Relevance Theory. In L. R. Horn \& G. L. Ward (Eds.), The handbook of pragmatics. Malden, MA; Oxford: Blackwell. http://dx.doi.org/10.1002/9780470756959. Accessed 2 May 2015

Teresa Guasti, M., Chierchia, G., Crain, S., Foppolo, F., Gualmini, A., \& Meroni, L. (2005). Why children and adults sometimes (but not always) compute implicatures. Language and Cognitive Processes, 20(5), 667-696. https://doi.org/10.108o/0169o96o44400025o

Tessler, M. H., Lopez-Brau, M., \& Goodman, N. D. (2017). Warm (for winter): Comparison class understanding in vague language. http://www.mit.edu/ tessler/papers/Tessler2o17-cogsci-final.pdf

Von Fintel, K.-U. (1994). Restrictions on quantifier domains. Doctoral Dissertations Available from Proquest. AAI9434544. https://scholarworks.umass.edu/dissertations/AAI9434544

Zinova, J., \& Filip, H. (2014). Scalar implicatures of Russian verbs. Formal approaches to slavic linguistics, 23 . 